\title{
Framework for Random Power Allocation of Wireless Sensor Networks in Fading Channels
}

\author{
Mohammed Elmusrati ${ }^{1}$, Naser Tarhuni ${ }^{2}$, Riku Jantti ${ }^{3}$ \\ ${ }^{1}$ Communication and Systems Engineering Group, University of Vaasa, Vaasa, Finland \\ ${ }^{2}$ Department of Electrical and Computer Engineering, Sultan Qaboos University, Muscat, Oman \\ ${ }^{3}$ Department of Communications and Networking, Aalto University, Helsinki, Finland \\ Email: moel@uwasa.fi, tarhuni@squ.edu.om,riku.jantti@aalto.fi
}

Received December 13, 2011; revised January 23, 2012; accepted February 11, 2012

\begin{abstract}
In naturally deaf wireless sensor networks or generally when there is no feedback channel, the fixed-level transmit power of all nodes is the conventional and practical power allocation method. Using random power allocation for the broadcasting nodes has been recently proposed to overcome the limitations and problems of the fixed power allocation. However, the previous work discussed only the performance analysis when uniform power allocation is used for quasi-static channels. This paper gives a general framework to evaluate the performance (in terms of outage and average transmit power) of any truncated probability density function of the random allocated power. Furthermore, dynamic Rayleigh fading channel is considered during the performance analysis which gives more realistic results that the AWGN channels assumed in the previous work. The main objective of this paper is to evaluate the communication performance when general random power allocation is used. Furthermore, the truncated inverse exponential probability distribution of the random power allocation is proposed and compared with the fixed and the uniform power allocations. The performance analysis for the proposed schemes are given mathematically and evaluated via intensive simulations.
\end{abstract}

Keywords: Power Allocation; Deaf Networks; Rayleigh Channels; Inverse Exponential Distribution

\section{Introduction}

There are many MAC protocols suggested for self-organized wireless sensor networks during the last two decades. All protocols are designed to achieve certain targets such as minimizing the MAC delay, maximizing the throughputs, minimizing the energy consumption, maximizing the network life-time, and many other objectives $[1,2]$. However, all these protocols assume that the sensor nodes have full listening (reception) capabilities. Therefore, the nodes can receive feedbacks and/or sense the environment (e.g., in CSMA/CA). The transmit power value is one of the radio resources which can be adjusted during the transmission. Efficient Power control schemes requires feedback channel between the receiver and the transmitter, where the receivers inform the transmitter about the channel quality in terms of different indicators such as the received signal-to-interference and noise ratio (SINR). This is known as closed-loop power control which is used mainly to mitigate distance and shadowing losses, fast fading, and most importantly to overcome the near-far effect in co-channel multiuser wireless communication systems (see e.g. [3-8]). However, when we discuss about naturally deaf sensor nodes, it becomes totally tricky how to optimize the transmission parameters (power, modulation-level, etc.). In such networks, the sensors' nodes are just transmitters (broadcasting) and do not have any receiving capabilities. Naturally deaf network is different than the temporary deafness of some networks because of channel fading or directive antennas. Generally, the transmission of deaf sensors can be eventbased or periodic. An example for both cases can be found the health structure monitor of the bridges. The even-based sensor will send the measured vibration of the structure if the vibration value exceeds a certain predefined value otherwise the sensors will stay silent. In periodic sensors, the sensors send the measured vibration of the structure periodically, for example every 10 seconds [9]. The transmit-only (TX) sensor nodes are much simpler and cheaper than the transceiver (TRX) sensor nodes. Moreover, it is observed that some TRX sensors consumes during the reception period more than $60 \%$ of the consumed energy during the transmission [1]. Hence, TX sensor nodes consume much less energy than TRX nodes. However, there are very few algorithms in the literature to improve the performance of deaf sensor nodes. Looking only to the power allocation for the TX sensor nodes, it is usually assumed that the nodes transmit with 
fixed power. This solution has many problems and limitations such as the near-far problem and the unnecessaryily power consumptions for good channel sensors.

New randomized power allocation strategy was suggested in [10], which proposed the use of uniformly distributed transmitter power levels to mitigate the near-far effect in congested systems without any channel feedback. That work was based on so called snapshot analysis approach and thus neglected the effects of the channel fading. The performance analysis of the uniform random power allocation in Rayleigh fading channel is evaluated in [11].

In this paper, a framework of the performance analysis for a general distributed random power allocation is introduced. The paper is organized as follows. In the next section, a description of the system model is given. For the logical information flow and for comparison purpose we introduce the system performance of the fixed power transmission in Section 3. In Section 4, a general treatment of the performance analysis of random power allocation algorithms is given. New empirical random power distribution is suggested in Section 5. Simulation results are shown in Section 6. Finally the paper conclusion is presented in Section 7.

\section{System Model}

In this paper we assume multiuser environment with broadcasting devices (sensor nodes) randomly distributed in certain region. We refer to the transmitters as terminals and sometimes as sensors. All terminals send their signals to one or more access points with CDMA multiple access method. Because of the lack of the feedback channels it is not possible to use CSMA/CA or any other protocols that require receiving capabilities in the sensor nodes. Multi-hop scenarios are not possible as well because of natural deafness. Every transmitter has different spreading code, however we do not assume that they are perfectly orthogonal at the access point.

We consider dynamic scenario, where the terminals or the access points may have mobility or the environment is highly dynamics. The transmitted signals arrive from sensors to the access point in multi-path manner without dominant path, in other words we assume Rayleigh channel. The time slot length is small enough to assume that second order effects such as shadow fading and distance based attenuation remain constant during the time duration of the time slot. Although the mean of the received signal is constant but the instant value of the received signal magnitude is random variable with Rayleigh probability density function. In case of Rayleigh fading which is considered here, the link gain, i.e. the fraction between received power and transmitted power becomes Exponential distributed random variable.

Time is assumed to be slotted such that slot duration is approximately the same as the coherence time of the channel. For instance in some sensor network applications, the duty cycle of the transmitters is low and thus the channel state in consecutive time slots allocated to single transmitter node become independent of each other.

Let $G$ denote the link gain between transmitter and receiver. In case of frequency-non-selective Rayleigh fading, it can be shown to follow the Exponential distribution with parameter $\lambda=1 / \bar{g}$ where $\bar{g}$ denotes the expected channel gain which depends on the distance based attenuation and shadow fading. Let $F_{G}(g)=1-e^{-\lambda g}$ denotes the cdf of the link gain $G$ and $f_{G}(g)=\lambda e^{-\lambda g}$ denotes its pdf.

Let $I$ and $\sigma_{n}^{2}$ denote the received interference and noise powers, respectively. Let $\gamma$ denotes the minimum required signal to interference and noise ratio (SINR) at the receiver. When the received SINR is less than $\gamma$, we assume that the receiver cannot decode the transmitted packet correctly. The required SINR depends on the utilized modulation and coding method and is out of the scope of the paper. The outage probability of sensor i, i.e. the probability that a packet error occurs, is given by

$$
\operatorname{Pr}\left\{\frac{G_{i} P_{i}}{I_{i}+\sigma_{n}^{2}} \leq \gamma\right\}
$$

where $G_{i}$ is the channel gain of sensor $i, P_{i}$ is the transmitted power from sensor $i$, and the interference term is given by

$$
I_{i}=\sum_{j \neq i}^{N} G_{j} P_{j}
$$

Note that in this model interference is treated as noise. The use of multi-user detection could be taken into account by scaling down the interference power $I$ by some factor $0<\theta<1$. However, this is not considered in this paper. We assume that the sensor in outage whenever its power at the access point is less than some threshold.

\section{Performance Analysis of Fixed Power Allocation}

In this section we analyze the system performance of fixed power transmission strategy. The results of this section is well known in the literature [6,11], however it is given here for the subject integrity and for comparison purposes. Moreover some intermediate results have been used in next sections. First we assume fixed average interference power (or single sensor scenario). In order to simplify our notation, let us define $\beta_{i}=\gamma\left(I_{i}+\sigma_{n}^{2}\right)$ Conditioned on $P_{i}=P$ the outage probability becomes

$$
\begin{aligned}
\operatorname{Pr}\left\{G_{i} P_{i} \leq \beta \mid P_{i}, \beta_{i}\right\} & =F_{G}\left(\frac{\beta_{i}}{P_{i}}\right) \\
& =1-e^{-\lambda \frac{\beta_{i}}{P_{i}}}
\end{aligned}
$$


For a given outage level $p_{\text {out }}$ we can find the corresponding fixed transmission power $P_{i}=P^{*}$ to be

$$
P^{*}=-\frac{\lambda \beta}{\ln \left(1-p_{\text {out }}\right)}
$$

In order to achieve low outage probability $P^{*}$ must be large. Consider now the case, where we have multiple sensors. In this case, $I$ cannot be treated as constant anymore, but rather as a random variable. Assume that all the $N$ transmitters are communicating with the same access point. In such case, the interference power at the receiver $I_{i}$ is given by (2). In this case we should average (4) over $\beta_{i}$.

From (2) we can deduce that the $p d f$ of the interference power is the $(N-1)$ fold convolution of exponential distribution $p d f$. This distribution is called Hypo-exponential distribution. Hence, the outage probability can be written as:

$$
\operatorname{Pr}\left\{G_{i} P_{i} \leq \beta_{i} \mid P_{i}\right\}=1-e^{\frac{\lambda_{i} \gamma \sigma_{n}^{2}}{P_{i}}} \int_{0}^{\infty} e^{-\frac{\lambda_{i} \gamma}{P_{i}} g} f_{I_{i}}(g) \mathrm{d} g
$$

We note that the integral in the above formula is in fact the moment generating function of the interference $M_{I_{i}}(t)=E\left\{e^{t I_{i}}\right\}$ where $t=-\frac{\lambda_{i} \gamma}{P_{i}}$. Let $Z_{i}=G_{i} P_{i}$ denotes the received power. In case of fixed transmission power, this still follows the exponential distribution with the following $p d f$ function

$$
f_{Z_{i}}(z)=\frac{\lambda_{i}}{P_{i}} e^{-\frac{\lambda_{i}}{P_{i}} z}
$$

The moment generating function of $Z_{i}$ can be easily found to be

$$
\begin{aligned}
M_{Z_{i}}(t) & =E\left\{e^{t Z_{i}}\right\} \\
& =\int_{0}^{\infty} e^{-t z} f_{Z_{i}}(z) \mathrm{d} z \\
& =\frac{1}{1-e^{t} \frac{P_{i}}{\lambda_{i}}}
\end{aligned}
$$

Now the moment generating function for the interference power \$I_i can be written as:

$$
\begin{aligned}
M_{I_{i}}(t) & =E\left\{e^{t \sum_{j \neq i} Z_{j}}\right\} \\
& =\prod_{j \neq i} E\left\{e^{t Z_{j}}\right\} \\
& =\prod_{j \neq i} M_{Z_{i}}(t)
\end{aligned}
$$

Let us revisit the outage probability (6). With the help of (10), (13), and (6) the outage probability of fixed power transmission is:

$$
\operatorname{Pr}\left\{G_{i} P_{i} \leq \beta_{i} \mid P_{i}\right\}=1-e^{-\frac{\lambda_{i} \gamma \sigma_{n}^{2}}{P_{i}}} M_{I_{i}}\left(-\frac{\gamma \lambda_{i}}{P_{i}}\right)
$$

$$
=1-e^{-\frac{\gamma \sigma_{n}^{2} \lambda_{i}}{P_{i}}} \prod_{j \neq i} \frac{1}{1+\frac{\gamma \lambda_{i} P_{j}}{\lambda_{j} P_{i}}}
$$

The outage probability shown in (15) is valid for any deterministic (fixed) or slowly changing power (power update rate is slower than the frame duration) transmission [12]. For randomly selected transmission power we need to average (6) over the probability density function of the transmitted power $f_{P}(p)$ as will be shown in the next section.

\section{General Distribution Random Power Allocation}

In this section we generalize the results of the previous section to the case of random transmitted power and analyzing the resultant performance in terms of outage probability and power consumption. Assume that sensor $i$ uses random transmitted power $P_{i}$ which has probability density function $f_{P}(p)$. First $\beta_{i}$ is assumed to be constant. This assumption could be justified for a single sensor-node scenario but not for multi sensor scenario. The outage probability in Rayleigh fading channel is given by

$$
\operatorname{Pr}\left\{G_{i} P_{i} \leq \beta_{i} \mid \beta_{i}\right\}=1-\int_{0}^{\infty} e^{-\lambda \frac{\beta_{i}}{p}} f_{P_{i}}(p) \mathrm{d} p
$$

where $f_{P_{i}}(p)$ is the $p d f$ of the random power allocation. Define a variable $x=1 / p$ it follows that $\mathrm{d} p=-\mathrm{d} x / x^{2}$, so (16) can be rewritten as

$$
\operatorname{Pr}\left\{G_{i} P_{i} \leq \beta_{i} \mid \beta_{i}\right\}=1-\int_{0}^{\infty} e^{-\lambda \beta_{i} x} f_{P_{i}}\left(\frac{1}{x}\right) \frac{\mathrm{d} x}{x^{2}}
$$

Note that

$$
f_{X}(x)=f_{P}\left(\frac{1}{x}\right) \frac{\mathrm{d} x}{x^{2}}
$$

is called the inverted distribution of $f_{P}(p)$ [13]. Theoretically any $p d f$ (should be truncated to be between some positive minimum and maximum values) could be used as $f_{P_{i}}(p)$. The probability density function that minimizes the outage probability (17) under mean power constraint can be shown to be the fixed power solution $P=$ $P_{\max }$, i.e., $f_{P_{i}}(p)=\delta\left(p-P_{\max }\right)$, where $\delta(p)$ is the dirac delta function. This property follows directly from Theorem 4.5.2 in [14]. However, in multi-user case finding the optimal distribution is not trivial. Randomization of the transmitted power implies randomization of the interference power which in turn can help to solve the nearfar problem.

Considering multi sensor scenario, where all sensors use the same power distribution $f_{P}(p)$. In this case $I_{i}$ should be considered as a random variable. We assume that $f_{P}(p)$ is selected so that the moment generating function for the received power $Z_{i}=G_{i} P_{i}$ is well-defined. 
That is, we assume that the $P_{i}$ has finite moments. In practice $P_{i}$ must be bounded in the interval $\left[P_{\min }, P_{\max }\right]$ from which this condition automatically follows. For a given $M_{Z_{i}}(t)$ we can find the corresponding $M_{I_{i}}(t)$ using (13) in Section 3. Let's first condition on $P_{i}$, then the outage probability can be found using (14) as,

$$
\operatorname{Pr}\left\{G_{i} P_{i} \leq \beta_{i} \mid P_{i}\right\}=1-e^{-\frac{\lambda_{i} \gamma \sigma_{n}^{2}}{P_{i}}} M_{I_{i}}\left(-\frac{\gamma \lambda_{i}}{P_{i}}\right)
$$

Thus the outage can be found using single integral,

$$
\operatorname{Pr}\left\{G_{i} P_{i} \leq \beta_{i}\right\}=1-\int_{0}^{\infty} e^{-\frac{\lambda_{i} \gamma}{p} \sigma_{n}^{2}} M_{I_{i}}\left(-\frac{\lambda_{i} \gamma}{P_{i}}\right) f_{P}(p) \mathrm{d} p
$$

Now taking into account that $P_{i}$ is bounded, hence, we can rewrite the above equation as

$$
\operatorname{Pr}\left\{G_{i} P_{i} \leq \beta_{i}\right\}=1-\int_{P_{\max }^{-1}}^{P^{-1}} e^{-\lambda_{i} \gamma \sigma_{n}^{2} x} M_{I_{i}}\left(-\lambda_{i} \gamma x\right) f_{p}^{t}\left(\frac{1}{x}\right) \frac{\mathrm{d} x}{x^{2}} \text { (20) }
$$

where $f_{p}^{t}(p)$ is the truncated version of the utilized probability density function. Without any loss of generality we will assume that $P_{\min }=0$ in remaining of this paper. Using (20), it is possible to evaluate the outage probability for arbitrary any truncated $p d f$. The average transmitted power is found by

$$
E[P]=\int_{0}^{P_{\max }} p f_{p_{i}}^{t}(p) \mathrm{d} p
$$

It is clear that randomizing the transmitted power results in an average power which is always less than $P_{\text {max }}$. This is one advantage of using random power over fixed power. By assuming random uniform distribution of the power between 0 and $P_{\max }$ the outage in this case is given by [11]

$$
\begin{aligned}
\operatorname{Pr}\left\{G_{i} P_{i} \leq \beta_{i}\right\}= & 1-\frac{1}{P_{\max }^{N} \lambda_{i}^{N-1} \gamma^{N-1}} \int_{P_{\max }^{-1}}^{\infty} \frac{1}{x^{N+1}} e^{-\lambda_{i} \gamma \sigma_{n}^{2} x} \\
& \times\left[\prod_{j \neq i} \lambda_{j} \ln \left(1+\frac{\gamma \lambda_{i} P_{\max }}{\lambda_{j}} x\right)\right] \mathrm{d} x
\end{aligned}
$$

Moreover, it is clear that in this case the average power will be $P_{\max } / 2$.

Now the most interesting point is to find the optimum distribution function which can achieve the following target:

$$
\begin{aligned}
& \text { Find } f_{p}^{t}(p) \text { such that } \\
& \qquad \begin{array}{c}
\max \sum_{i} \int_{P_{\max }^{-1}}^{P_{\min }} e^{-\lambda_{i} \gamma \sigma_{n}^{2} x} M_{I_{i}}\left(-\lambda_{i} \gamma x\right) f_{p}^{t}\left(\frac{1}{x}\right) \frac{\mathrm{d} x}{x^{2}} \\
\text { S.T. } \int_{0}^{P_{\max }} p f_{p_{i}}^{t}(p) \mathrm{d} p \leq \hat{P}
\end{array}
\end{aligned}
$$

It is possible also to change the order, i.e., minimize the average power subject to certain outage. So far, we are not sure if the above optimization problem is solvable or not. However, we leave it as an open problem for further research. Nevertheless, a random power distribution is proposed based on empirical assumptions as shown in the next section.

\section{Inverted Exponential Distribution}

Since the received power has an exponential distribution when the transmitted power is fixed, we propose random transmitted power with inverted exponential distribution to mitigate the fading channel. This selection was not based on any optimization criteria. However, this empirical selection shows few features and advantages over the uniform distribution of the power allocation as will be discussed in the next section. The inverted exponential distribution is given by

$$
f_{P}(p)=\frac{\alpha}{p^{2}} e^{-\frac{\alpha}{p}}, p \geq 0
$$

where $\alpha$. The cumulative probability density function is given by

$$
F_{P}(p)=e^{-\frac{\alpha}{p}}
$$

The outage probability for single sensor scenario utilizing random power allocation with inverted exponential distribution can be computed using (16) and (25) such as

$$
\operatorname{Pr}\left\{G_{i} P_{i} \leq \beta_{i} \mid \beta_{i}\right\}=\frac{\lambda_{i} \beta_{i}}{\alpha+\lambda_{i} \beta_{i}}=1-\frac{\alpha}{\alpha+\lambda_{i} \beta_{i}}
$$

From (27) the relation between $p d f$ parameter $\alpha$ and the outage is given by

$$
\alpha=\frac{1-p_{\text {out }}}{p_{\text {out }}} \lambda_{i} \beta_{i}
$$

Thus the smaller $p_{\text {out }}$ the larger $\alpha$, for zero outage $\alpha \rightarrow \infty$.

The probability that the power allocation exceeds some maximum value $P_{\max }$ is given by

$$
\operatorname{Pr}\left\{P \geq P_{\max }\right\}=1-F_{P}\left(P_{\max }\right)=1-e^{\left(\frac{1-p_{\text {out }}}{p_{\text {out }}}\right) \frac{\lambda_{i} \beta_{i}}{P_{\max }}}
$$

In order to avoid using excessive power, the power distribution should be truncated to be between 0 and $P_{\max }$. The truncated inverse exponential distribution is given by:

$$
f_{P}(p)=\left\{\begin{array}{cc}
\frac{\alpha}{p^{2}} e^{-\frac{\alpha}{p}} & 0 \leq p \leq P_{\max } \\
0 & \text { otherwise }
\end{array}\right.
$$

In this case, we have

$$
\operatorname{Pr}\left\{G_{i} P_{i} \leq \beta_{i} \mid \beta_{i}\right\}=1-\frac{\alpha}{\alpha+\lambda_{i} \beta_{i}} e^{-\frac{\lambda_{i} \beta_{i}}{P_{\max }}}
$$

The outage in this case depends on the value of $P_{\max }$ as 
well as the value of $\alpha$. We can now find the required peak power for given outage level $p_{\text {out }}$

$$
P_{\max }=-\frac{\lambda_{i} \beta_{i}}{\ln \left(1+\frac{\lambda_{i} \beta_{i}}{\alpha}\right)+\ln \left(1-p_{\text {out }}\right)}
$$

for

$$
\alpha>\frac{1-p_{\text {out }}}{p_{\text {out }}} \lambda_{i} \beta_{i}
$$

We note that as $\alpha \rightarrow \infty, P_{\max } \rightarrow P^{*}$ given in (5). Thus in single sensor scenario, the random power allocation requires higher peak transmit power than the fixed allocation to achieve same average outage. This result can be generalized for any truncated distribution function. Figure 1 shows the truncated inverted exponential distribution for different values of $\alpha$. It shows that as $\alpha$ increases the random power becomes more close to $P_{\max }$. From (30) when $\alpha \rightarrow \infty$ the distribution tends to be fixed power at $P_{\max }$, i.e., $f_{P_{i}}(p)=\delta\left(p-P_{\max }\right)$.

The average transmitted power of using truncated inverted exponential distribution can be computed using (21) and (30) as

$$
\begin{aligned}
E[P] & =e^{\frac{\alpha}{P_{\max }}} \int_{0}^{P_{\max }} \frac{\alpha}{p} e^{-\frac{\alpha}{p}} \mathrm{~d} p \\
& =\alpha e^{\frac{\alpha}{P_{\max }}} \int_{\frac{\alpha}{P_{\max }}}^{\infty} \frac{1}{x} e^{-x} \mathrm{~d} x \\
& =\alpha e^{\frac{\alpha}{P_{\max }}} E_{1}\left(\frac{\alpha}{P_{\max }}\right)
\end{aligned}
$$

where

$$
E_{n}(x)=\int_{1}^{\infty} t^{n} e^{-x t} \mathrm{~d} t=x^{n-1} \int_{x}^{\infty} t^{-n} e^{-t} \mathrm{~d} t
$$

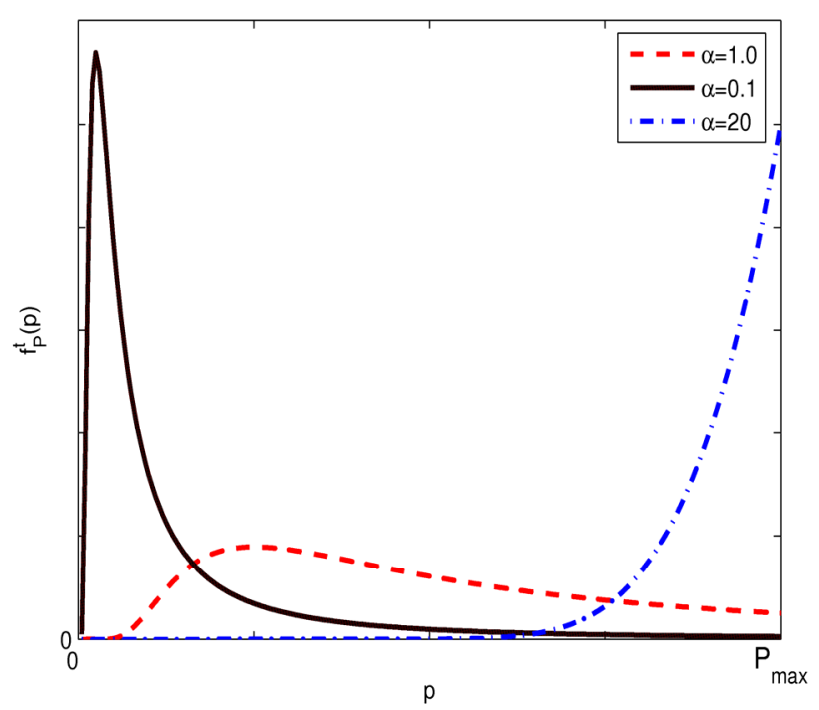

Figure 1. Truncated inverted exponential $p d f$. is called the exponential integral of order $n$. Note that $E_{n}(x) \geq 0$ for $x \geq 0$. The asymptotic expansion of $E_{n}(x)$ can be written as

$$
E_{n}(x)=\frac{e^{-x}}{x}\left(1-\frac{n}{x}+\frac{n(n+1)}{x^{2}}+\cdots\right)
$$

Figure 2 shows the relation between the average transmitted power and $\alpha$ when $P_{\max }=1$.

The probability of outage in multi-user environment where all transmitters utilize truncated inverted exponential distribution is discussed next. Without loss of generality we assume that all transmitters use same distribution parameter $\alpha$. This assumption is practical since no information is available for transmitters about neither their channel quality nor their locations. We will discuss the influence of the selection of $\alpha$ on the system performance in the next section. The cdf of the received power $Z_{i}=G_{i} P_{i}$, denoted by $F_{Z_{i}}(z)$, is given by (31) by replacing $\beta_{i}$ by $z$. Let us now derive the moment generating function $M_{Z_{i}}(t)$ using $F_{Z_{i}}(z)$. We first note that $F_{Z_{i}}(0)=0$ and $F_{Z_{i}}(z) \rightarrow 1$ as $z \rightarrow \infty$. Let $s=-t>0$. Using integration by parts we get,

$$
\begin{aligned}
M_{Z_{i}}(-S) & =\int_{0}^{\infty} \frac{\mathrm{d}}{\mathrm{d} z} F_{Z_{i}}(z) e^{-s z} \mathrm{~d} z \\
& =-\left.s F_{Z_{i}}(z) e^{-s z}\right|_{0} ^{\infty}-s \int_{0}^{\infty} F_{Z_{i}}(z) e^{-s z} \mathrm{~d} z \\
& =1-s \int_{0}^{\infty} \frac{\frac{\alpha}{\lambda_{i}}}{\frac{\alpha}{\lambda_{i}}+z} e^{-\left(s+\frac{\lambda_{i}}{P_{\max }}\right) z} \mathrm{~d} z
\end{aligned}
$$

Now from [15], we can find that,

$$
\int_{0}^{\infty} \frac{1}{a+t} e^{-s t} \mathrm{~d} t=e^{a s} E_{1}(a s), a>0
$$

Hence,

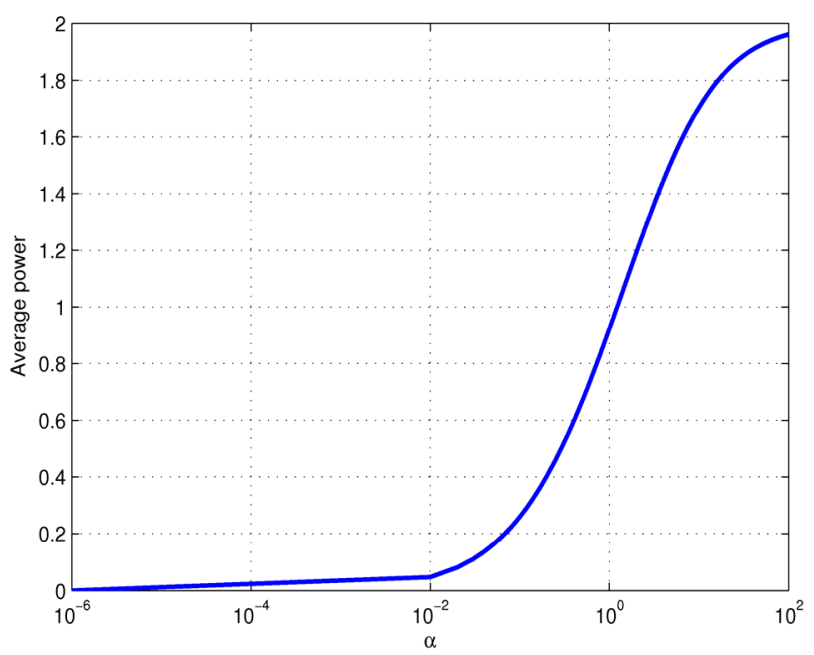

Figure 2. Average transmitted power with respect to $\alpha$. 


$$
M_{Z_{i}}(t)=1+t \frac{\alpha}{\lambda_{i}} e^{\frac{\alpha}{\lambda_{i}}\left(\frac{\lambda_{i}}{P_{\max }}-t\right)} E_{1}\left(\frac{\alpha}{\lambda_{i}}\left(\frac{\lambda_{i}}{P_{\max }}-t\right)\right)
$$

Once again we can solve the moment generating function for the interference power $M_{I_{i}}(t)$ using (13). From the general outage probability formula (20) and (30) we can find the mathematical formula for the outage probability in case on inverted exponential power allocation such as,

$$
\begin{aligned}
& \operatorname{Pr}\left\{G_{i} P_{i} \leq \beta_{i}\right\}=1-\alpha e^{\frac{\alpha}{P_{\max }}} \int_{P_{\max }^{-1}}^{\infty} e^{-\left(\lambda_{i} \gamma \sigma_{n}^{2}+\alpha\right) x} \\
& \quad \times\left[\prod_{j \neq i}\left(1-\gamma \alpha \frac{\lambda_{i}}{\lambda_{j}} x e^{\left(\frac{\alpha}{P_{\max }}+\frac{\alpha \gamma \lambda_{i} x}{\lambda_{j}}\right)} E_{1}\left(\frac{\alpha}{P_{\max }}+\frac{\alpha \gamma \lambda_{i} x}{\lambda_{j}}\right)\right] \mathrm{d} x\right.
\end{aligned}
$$

It can be shown that when $\alpha \rightarrow \infty$ the above equation will be reduced to (15), the case when the transmitted power from sensors are fixed and identical.

\section{Numerical Results}

Different aspects will be evaluated in this section. In the first part we validate Equation (43) by conducting extensive Monte-Carlo simulations. And in the second part we test the performance of the random truncated exponential allocation algorithm for different scenarios and compareing it with fixed and random uniform power allocation schemes. All simulations are carried out for Rayleigh channels with an average of $d^{4}$, where $d$ is the distance between the sensor and the access point. The background white noise power is fixed at $-70 \mathrm{dBm}$. The multiple access method for all nodes is CDMA and the processing gain is $27 \mathrm{~dB}$. The target $E_{b} / N_{o}$ is $7 \mathrm{~dB}$. It means that the minimum required SNR is about 0.01 for all sensors.

Figure 3 shows the outage with respect with number of sensors using exact formula (43) and Monte-Carlo simu-

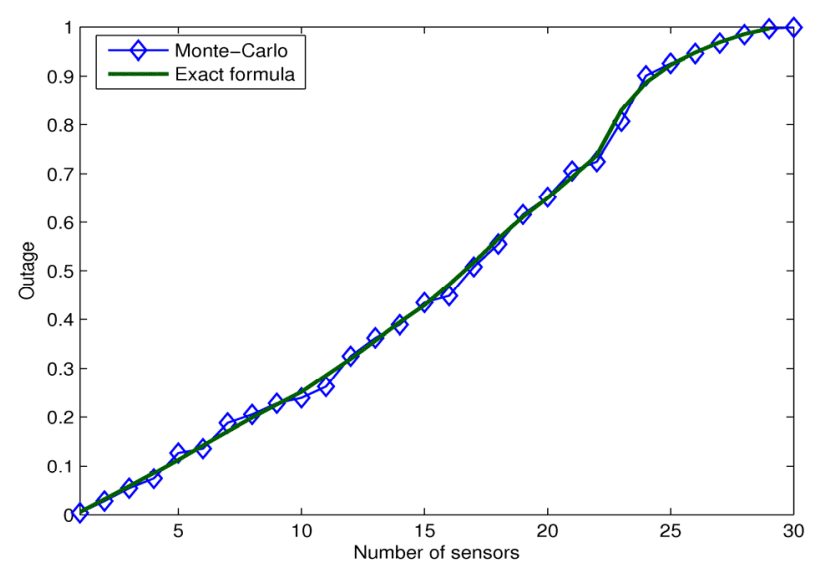

Figure 3. Average outage using the exact formula and Monte Carlo simulations. lation. The simulation has been carried out by generating 30 sensors with randomly distributed distance from the access-point from 20 up to 150 meters. The outage is calculated by counting the total number of packets where the SINR is less than 0.01, and then dividing this number by the total number of sensors at that stage. This procedure repeated 1000 times to obtain a reliable measure for the outage. The sensors are increased one by one in ascending manner, i.e., the first sensor is the nearest to the access point and the second is the next further one and so on. The derived formula gives directly the probability of outage by substituting the average channel losses of sensors in (43).

To compare the performance of inverted exponential random power (IERP) allocation with fixed and uniform power, we repeat the previous simulation including other power allocation methods. Figure 4 shows the outage for all cases at $\alpha=0.5$, maximum power of $1 \mathrm{Watt}$ and the uniform distribution is truncated between 0 and 1 . In terms of outage, it is clear that IERP allocation outperforms the uniform power when the number of sensors is less than about 22 sensors. For larger network size the IERP allocation outperform the fixed power allocation and becomes very close to the uniform. In terms of power consumption the IERP consumes less average power than both other methods. The average required power is 1, 0.5, and 0.46 Watt for fixed, uniform and IERP methods, respectively. This result shows one benefit of using IERP allocation over fixed or uniform allocation. It gives less average outage for large network size than the fixed power allocation at lower average power consumption. Figure 5 shows the average outage of worst sensor with respect to the minimum required SINR for different values of $\alpha$, i.e., with different average power values.

In this scenario we mean by worst sensor is the one which is located on the cell boarder, i.e., at 150 meters. However, because of the fading behavior it is not neces-

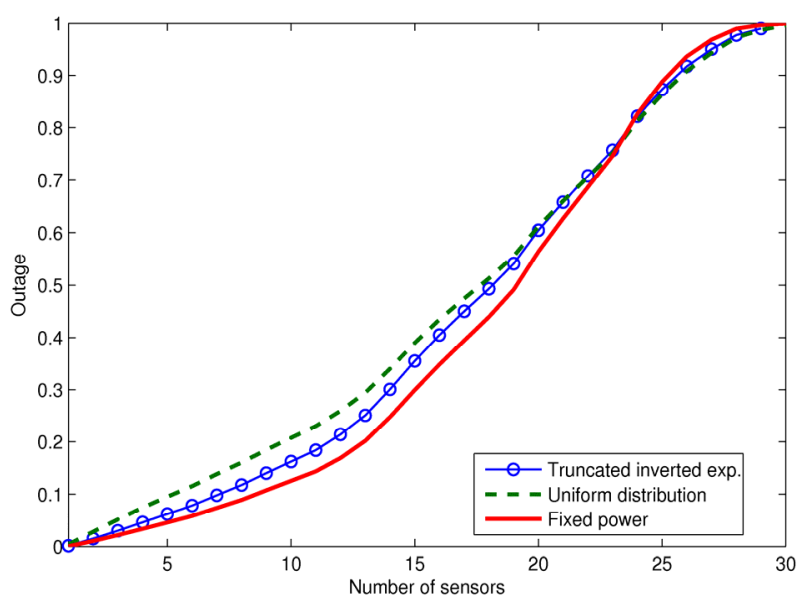

Figure 4. The average outage using different power allocation methods. 


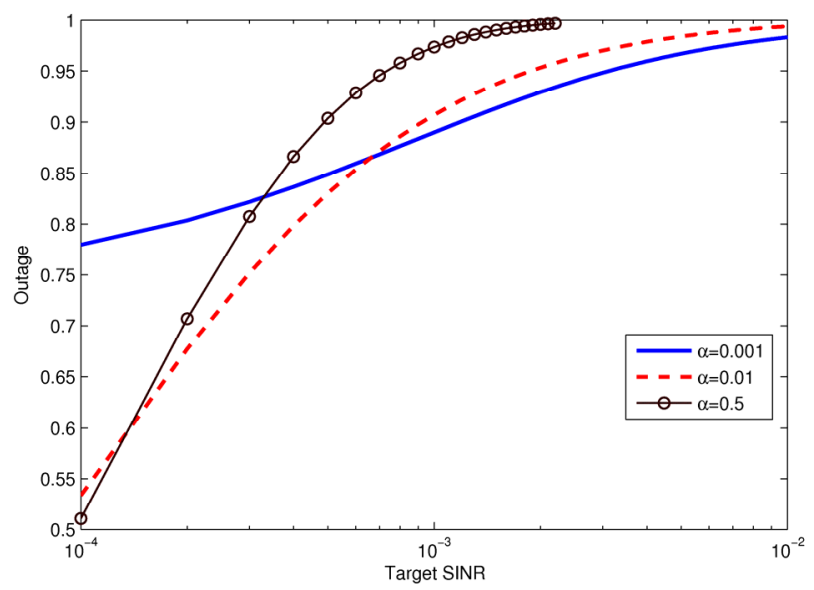

Figure 5. The average outage of worst sensor for different values of $\alpha$.

sary that this sensor has all time the worst channel. The figure shows that as the $\alpha$ becomes smaller, the outage of the worst sensor reduces, and it gets a better chance to access the network. The reason is clearly because of reducing the interference coming from closer sensors by reducing their average transmitted power. However, reducing $\alpha$ has a negative impact on the average outage of all sensors as shown in Figure 6. Figure 6 shows also the average outage when using uniform and fixed power allocations. This last simulation has been carried out for 15 sensors; other simulation parameters are as before. The average power used for IERP increases with $\alpha$. At $\alpha=0.61$ the average power is 0.5 Watt which is the same average power of uniform allocation. However, the IERP archives the same outage as uniform allocation at $\alpha \approx 0.165$ which means average power of 0.26 Watt. This result is rather interesting where smaller average outage can be achieved at less average power. The fixed power scenario all the time has the highest average power consumption (1 Watt).

\section{Conclusion}

The main aim of this paper is to introduce a general framework analysis for random power allocation methods in Rayleigh fading channels. Simple mathematical procedure has been given to analysis the system performance when using any arbitrary truncated random power distribution. We extend our work by proposing the truncated inverted exponential probability density function (IERP) for the random power. Mathematical representation of the outage as well as the average transmitted power is given. IERP shows many advantages over fixed as well as over random uniform power allocations. At small network size IERP gives less average outage than the uniform power allocation. For large network size IERP gives less outage than the fixed power allocation method. At the same outage the IERP needs less average power

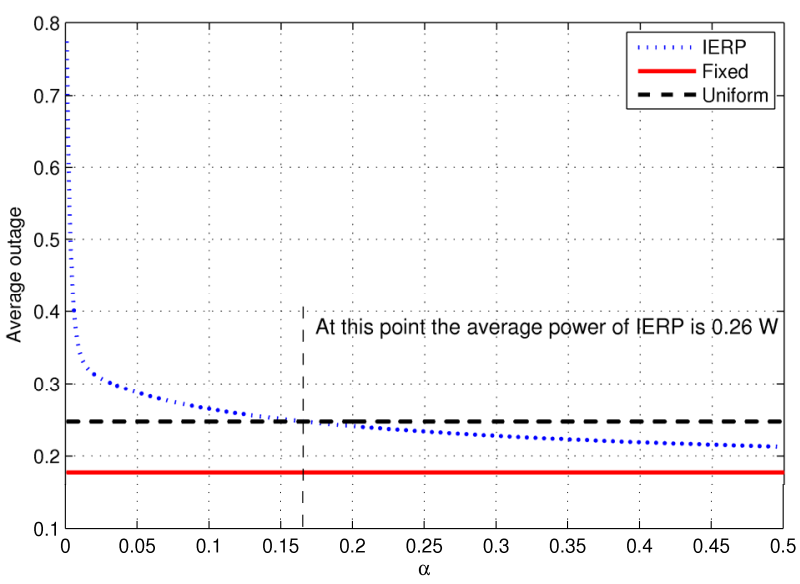

Figure 6. The average outage sensors versus $\alpha$.

than the uniform distribution. The required power depends on the network size and nodes spatial distribution. Another good advantage of the IERP that it has only one parameter to tune, this reduces the difficulties of optimization over multi-dimensions. However, according to the intensive mathematical and simulation analysis in this work, it was obvious that using random power allocation will not improve the outage performance considerably when considering realistic fading channels. However, it is clearly reduce the average power consumption. Nevertheless, the topic is still open for several research paths such as find the optimum power distribution (if any), diversity gain analysis with random power allocation, and simplify the problem by using discrete power values with predefined probability.

\section{REFERENCES}

[1] A. Bachir, M. Dohler, T. Watteyne and K. Leung, "MAC Essentials for Wireless Sensor Networks," IEEE Communications Surveys \& Tutorials, Vol. 12, No. 2, 2010, pp. 222-249.

[2] J. Haapola, "Evaluating Medium Access Control Protocols for Wireless Sensor Networks,” Ph.D. Thesis, University of Oulu, Oulu, 2010.

[3] J. Zander, "Performance of Optimum Transmitter Power Control in Cellular Radio Systems," IEEE Transactions on Vehicular Technology, Vol. 41, No. 1, 1992, pp. 57-62.

[4] R. D. Yates, "A Framework for Uplink Power Control in Cellular Radio Systems,” IEEE Journal on Selected Areas in Communications, Vol. 13, No. 7, 1995, pp. 1341-1347.

[5] M. Elmusrati, R. Jäntti and H. N. Koivo, "Multi-Objective Distributed Power Control Algorithm for CDMA Wireless Communication Systems,” IEEE Transactions on Vehicular Technology, Vol. 56, No. 2, 2006, pp. 779-788.

[6] A. J. Viterbi, A. M. Viterbi and E. Zehavi, "Performance of Power Controlled Wideband Terrestrial Digital Communication," IEEE Transactions on Communications, Vol. 41, No. 4, 1993, pp. 559-569. doi:10.1109/26.223780

[7] J. H. Kim, S. J. Lee, Y. W. Kim, M. Y. Chung and D.K. 
Sung, "Performance of Single-Bit Adaptive Step-Size Closed-Loop Power Control Scheme in DS-CDMA Systems," IEICE Transactions on Communications, Vol. E81-B, No. 7, 1998, pp. 1548-1552.

[8] M. Rintamäki, H. Koivo and I. Hartimo, “Adaptive ClosedLoop Power Control Algorithms for CDMA Cellular Communication Systems," IEEE Transactions on Vehicular Technology, Vol. 53, No. 6, 2004, pp. 1756-1768. doi:10.1109/TVT.2004.836937

[9] M. Bocca, et al., “A Synchronized Wireless Sensor Network for Experimental Modal Analysis in Structural Health Monitoring," Computer-Aided Civil and Infrastructure Engineering, Vol. 26, No. 7, 2011, pp. 483-499.

[10] T.-S. Kim and S.-L. Kim, "Random Power Control in Wireless ad hoc Networks," IEEE Communications Letters, Vol. 9, No. 12, 2005, pp. 1046-1048. doi:10.1109/LCOMM.2005.1576583

[11] M. Elmusrati, N. Tarhuni and R. Jäntti, "Performance Ana- lysis of Random Uniform Power Allocation for Wireless Networks in Rayleigh Fading Channels," European Transactions on Telecommunications, Vol. 20, No. 4, 2009, pp. 457-462.

[12] M. Elmusrati, N. Tarhuni and R. Jantti, "Performance Analysis for Wireless Deaf Sensor Networks in Fading Channels” IEEE 65th Vehicular Technology Conference, Dublin, 22-25 April 2007, pp. 189-182.

[13] E. L. Lehmann and J. P. Shaffer "Inverted Distributions," The American Statistician, Vol. 42, No. 3, 1988, pp. 191194. doi:10.2307/2684999

[14] J. S. Rustagi, "Variational Methods in Statistics,” Academic Press, London, 1976.

[15] M. Abramowitz and I. A. Stegun, "Handbook of Mathematical Functions with Formulas, Graphs, and Mathematical Tables," US Department of Commerce, 1979. http://www.knovel.com/knovel2/Toc.jsp?BookID=528\& VerticalID $=0$ 